\title{
An Approach to Determine Coordinate Transformation Parameter for Nepal GPS Network
}

\author{
Kalyan Gopal Shrestha \\ Deputy Director General, Survey Department \\ kalyanshrestha@gmail.com
}

\begin{abstract}
The Surveying and Mapping community now has the benefit of 3-dimensional coordinates at the centimeter level, through the Global Positioning System (GPS). The reference frame for GPS, World Geodetic System of 1984 (WGS84), within which a user ascertains these coordinates is essentiallygeocentric. All coordinated data and mapping in Nepal are based on a non-geocentric coordinate system known as the Everest Datum of 1830. This paper tries to present a practical approach to define transformation parameters between the two coordinate systems for Nepal.
\end{abstract}

\section{Beginning of geodesy in Nepal}

In Nepal, requirement of good maps and control points were not understood until the end of 50's. The surveying and mapping activities in Nepal before 1945 were limited to the preparation of parcel survey covering a small area. After 1945, actual works can be said to begin. Survey of India established a series of triangulation chains in Nepal under the Colombo plan agreement for the surveying and mapping activities in Nepal. The points of the chains were tied to the great trigonometrical networks in India bordering Nepal for $1 "$ = 1 mile topographical mapping of the country. In 1970, establishing geodetic controls were felt essential and Geodetic Survey Branch (GSB), then Trigonometrical Survey Branch was established under Survey Department. Geodetic Survey Branch started establishing second, third and fourth order control points based on those Survey of India control points.

\section{Conventional survey practice}

Traditionally, the geodetic surveying operations for precise positioning of points on the surface of the Earth for the purpose of mapping the region were very complex, laborious and time-consuming processes. The positioning, or provision of "control points", which controlled the geometric properties and the scale of the map, was carried out using ground measurements of lengths and angles, using Theodolites and chains/tapes, which were later replaced by Electronic Distance Measuring instruments (EDMs). Both these terrestrial methods demanded extensive field work, involving large manpower, expenditure, efforts and time; thus making the geodetic surveying and mapping process slow and tedious. In order to ensure inter-visibility between survey stations, towers were often constructed and beacons were erected. Survey Department of Nepal took two decades of labour-extensive work for the geodetic surveys of the country using these classical techniques.

The then Trigonometrical Survey Branch established Fundamental trigonometric station, Fundamental Baseline and Astronomical Observatory at Nagarkot in 1977. Same year Leveling Division started precise First Order Leveling network in the country.

A team of Czechoslovakian astronomical experts established 7 Laplace stations with 14 Azimuth stations with the assistance of UNDP in 1977. The establishment and triangulation survey of First order control net with the help of Ministry of Defense of the United Kingdom (UK) was completed in 1986. This First order Triangulation Network in Nepal is one major step in geodetic history of the country. This has fulfilled the necessity of the requirement of a geodetic datum in the country. This net is consistent with international standards of the base defined by Doppler Satellite Observations. This network with 68 stations, of which 16 stations were observed by Doppler technique, is distributed entirely all over the country except high Himalayan part.

The challenging task of carrying out geodetic surveys for mapping the mountainous nation, with diverse terrains, from the snowy peaks of Himalayas to the Tarai plains, was completed by the dedicated geodesists and surveyors of Nepal over the years. The completion of the Triangulation Survey of Nepal, with over 4000 geodetic stations (including the first, second and third orders) was made possible by the dedicated efforts of these surveyors.

The geodetic survey control net works of NEPAL, including the Triangulation, high precision leveling, 
Laplace stations, gravimetric stations, the Doppler survey stations and Global Positioning System (GPS) stations, form one extensive and precise geodetic networks. These networks also form the foundation of the precise mapping of the entire country.

\section{Introduction of space geodetic techniques}

The positioning of precise coordinates of control points for mapping purpose, by Triangulation, one of groundbased methods, continued till the introduction of the space geodetic techniques. Global Positioning System (GPS), with its economy, low cost, versatility, and ease of operation, has become the most preferred positioning method for geodetic surveying, within few years of its launch. The introduction of GPS has truly revolutionized the field of modern surveying and mapping in the whole world over last two decades. GPS is today being used for a wide spectrum of applications in NEPAL.

Nepalese Survey Department (SD) ushered into GPS age only in 1988 even though GPS has been operational in USA since 1970s. For the first time, Topographical Survey Branch of SD bought a pair of WM102 GPS receivers and used in Nepal-India boundary survey. During the Lumbini Zone Topographic Mapping Project, funded by Government of Japan, Trimble receivers were used for the topographical mapping works in 1988. During 1992-95 Eastern and Western Nepal Topographic Mapping Project, funded by Finland Government, established a number of GPS stations. Eastern Nepal Topographic Mapping Project (ENTMP) established 101 stations while Western Nepal Topographic Mapping Project (WNTMP) established 127 stations covering even the high Himalayan region and surveying were done with Ashtech receivers. Most of the baselines of these project-surveyed stations are more than $50 \mathrm{kms}$.

The collaborative project of the University of Colorado and Massachusetts Institute of Technology (USA) brought Trimble and Ashtech receivers and set up new GPS stations at 29 different locations covering Nepal. They captured satellite data from those stations in 1991. In 1996 the University again tracked continuous data from those stations for few days for crustal movement studies.

GPS field works at different periods for different purposes with different accuracies have provided chance to many staffs of Survey Department to be acquainted with different types of GPS receivers. The triangulation network was surveyed using theodolite and distance measuring instruments. The network distributed only over non-high Himalayan parts of the country. But with the introduction of Global Positioning System (GPS) the network extended even to high Himalayan region.
Geodetic Survey Branch in 1994 setup a separate GPS section with couples of Leica made WILD GPS system200 receivers to densifying, and strengthening the higher order National Geodetic network. Currently, this branch has GPS system-500 receivers too. Now a day's GPS is the main techniques for the extension of higher order controls in Nepal.

\section{Everest \& WGS-84 datum}

Nepal has been using Everest Spheroid 1830 as national datum for mapping controls. The coordinates (in Geographic or Cartesian) determined by GPS are not compatible with local coordinate values and must be transformed to National Coordinate System of Nepal.

The terrestrial reference system used by the U.S. Department of Defense (DoD) for GPS positioning is the World Geodetic System 1984 (WGS-84). WGS-84 is a global geocentric coordinate system based on Doppler observations of the TRANSIT satellite system

\subsection{Datum transformation parameters}

Coordinate transformation is the mathematical procedure to establish a geometrical relationship between a source coordinate system (local or image coordinate system) and a target coordinate system (world or object coordinate system). This procedure estimates the transformation parameters using a set of control points measured in the two coordinate systems. The process of mathematically converting positions from one datum to the other is known as datum transformation. Datum transformation parameters define functional relationship between two reference frames.

\subsection{Transformation procedure}

GPS transformation can be carried out in two ways:-

Using published transformation parameters.

Through the determination of the appropriate transformation parameters.

So far, there is no accurate and officially published set of transformation parameters in Nepal. There are a number of ways to mathematically transform positions from one datum to another, but they all require "common points".

\subsubsection{Selection of common points}

Common points are surveyed points that have known positions in terms of both the local and the global datum. For determining transformation parameters between Everest \& WGS-84 datum, evenly distributed common points throughout the country are selected for GPS observations. The points were chosen in such a manner that it represent a good sample of good relationship between 
the local and global datum. The achievable accuracy of the datum transformation will be determined by the number, distribution and accuracy of these common points and the transformation technique adopted. Generally speaking, the greater the accuracy required, the more common points are needed. Since Indian triangulation network comprised of independently adjusted series with different level of accuracy it is unlikely to get a single set of transformation parameter with desirable accuracy for the entire country.

\subsubsection{GPS observations and data processing}

A comprehensive observation plan is being run to ensure the smooth operation of field programme. Continuous GPS observations for 12 hrs at each station were carried out with a sampling rate of 15 seconds. Since the distance between two consecutive stations was more than $100 \mathrm{~km}$ nearly 10,000 sq. km area has to be covered in one exercise involving 6 GPS receivers. The entire field observation work is evenly distributed between independent teams, each comprised of experienced personnel.

After completion of field work the observed data are processed with precise ephemerides using comprehensive software. WGS-84 coordinates of each observed station were determined by processing the observed GPS data in combination with reference stations during the processing operation. The computed coordinates along with known Everest coordinates of each observed point formed the data set for estimation of transformation parameters.

\subsubsection{Transformation models}

There are a number of ways of defining the relationship between one reference system and another. The choice of the most appropriate network transformation model is influenced by such factors as:-

- Whether the model be applied to a small area, or over a large region.

- Whether one (or both) networks have significant distortions.

- The accuracy required.

The most general and simple transformations is the affine transformation. An affine transformation transforms straight lines to straight lines and parallel lines remain parallel but the size, shape, position, and orientation of lines in a network may change.

Several mathematical models have been developed which describe the functional relationship between pairs of three dimensional coordinates. Two mathematical transformation models: The Bursa-Wolf transformation model and The Molodensky-Badekas model are mostly common and tested to find best solution. The both models are explained below.

\subsubsection{The Bursa-Wolf transformation model:} In the geodetic context, the general transformation model in eqn (1) is often referred to as the Bursa-Wolf model. The 3-D similarity transformation model relating coordinates of points in the $X_{B} Y_{B} Z_{B}$ network to coordinates in the $\mathrm{X}_{\mathrm{A}} \mathrm{Y}_{\mathrm{A}} \mathrm{Z}_{\mathrm{A}}$ network is:

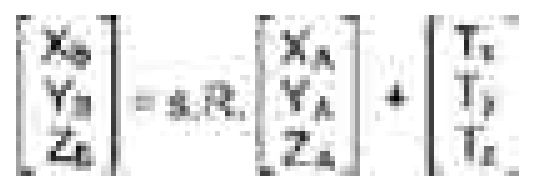

where $\mathrm{s}$ is the scale factor and $\mathrm{R}$ is a $3 \times 3$ orthogonal rotation matrix (eqn (4)). Note that there are seven parameters: three rotation angles, three translation components and one scale factor. The translation terms $T_{x}, T_{y}, T_{z}$ are the coordinates of the origin of the $\mathrm{X}_{\mathrm{A}} \mathrm{Y}_{\mathrm{A}} \mathrm{Z}_{\mathrm{A}}$ net in the frame of the $\mathrm{X}_{\mathrm{B}} \mathrm{Y}_{\mathrm{B}} \mathrm{Z}_{\mathrm{B}}$ net.

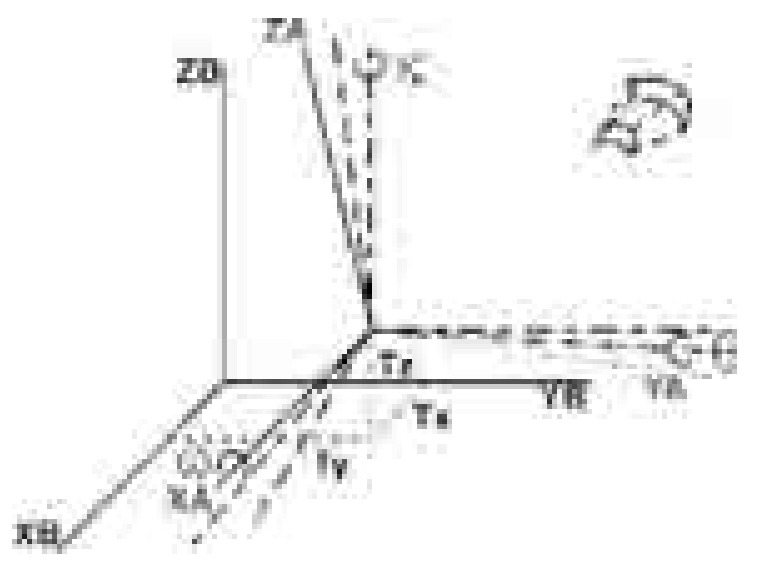

The seven parameter 3-D similarity transformation model.

\subsubsection{The Molodensky-Badekas model:}

An alternative model is the Molodensky-Badekas model, the equation of this model is as follows:-

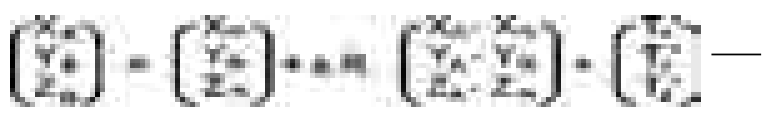

where $\mathrm{X}_{\mathrm{m}}=\bar{E}_{\mathrm{X}_{\mathrm{Ai}}} / \mathrm{n}, \quad \mathrm{Y}_{\mathrm{m}}=\bar{\Sigma}_{\mathrm{Y}_{\mathrm{Ai}}} / \mathrm{n}, \quad \mathrm{Z}_{\mathrm{m}}=\bar{\Sigma}_{\mathrm{Z}_{\mathrm{Ai}}} / \mathrm{n}$ are the coordinates of the centroid of the network. Alternatively $X_{m}, Y_{m}, Z_{m}$ may be selected to be the coordinates of one of the points in network A. Although the translation parameters are different, the rotation matrix and the scale factor are the same as for the Bursa-Wolf model. 


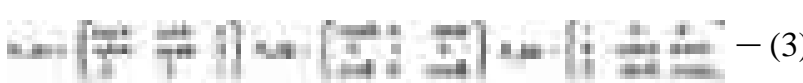

The rotation matrices about the $\mathrm{X}$-, $\mathrm{Y}$-, and $\mathrm{Z}$-axes are:

The most common combined rotation matrix is: $\mathrm{R}=\mathrm{R}_{\mathrm{z}}$ ( $\mathrm{K}$ ). $\mathrm{R}_{\mathrm{y}}(\mathrm{D}) \cdot \mathrm{R}_{\mathrm{x}}(\mathrm{I})$, leading to:

(4)

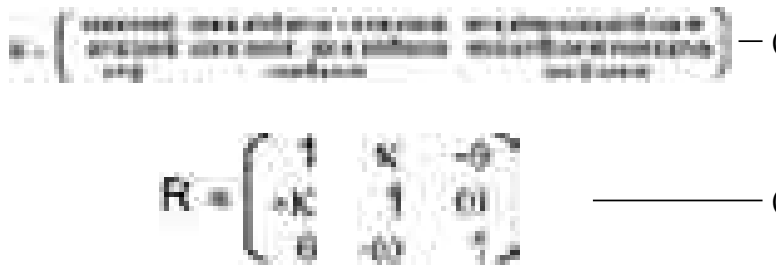

For small rotations this matrix may be approximated by:

where $\mathbf{E}, \mathbf{E}$, and $\mathbf{k}=$ are the rotation angles in radians about the X-, Y-, and Z-axes respectively.

The Bursa-Wolf transformation model is the most popular and effective one in most of the countries as it does not require local origin coordinates to be maintained along with the transformation parameter equation. The simplicity of the Bursa-Wolf Transformation model is another reason of applying it for our transformation.

\section{Features of survey of India (SOI) triangulation network (1946-63):}

According to the report and chart of triangulation control points survey completed during 1946-63 made available to Geodetic Survey Branch by Survey of India, following features can be identified. Nine independent series of triangulation chains were established to make a framework of control for the topographical mapping (1" = 1 mile) of Nepal. The average and maximum triangular errors in nine different series are given below.-
The remaining triangulation network inside Nepal was based on stations of the above series with average triangular error 5 seconds and maximum triangular error 30 seconds. (Source: SOI Report, 27 June 1977)

The table- 1 shows the triangulation series do not form a single network and are not homogeneous. The triangulation series named F(main), E (a) and E (b); the closing error in these chain were not adjusted where as E(c) and F (sub) the chains are left unadjusted. The reason behind this could be perhaps the starting and closing of the triangulation chain was not done in higher order stations or may be misclosure were exceeding this tolerance or the accuracy obtained was enough for the topographical mapping project.

\section{New network and parameters}

From the table 1 it can be suspected that a single set of transformation parameters for the entire country may not give the level of accuracy required due to inconsistency and irregularity in the triangulation network. The conventional triangulation survey measurement is suffered from accumulation of scale errors whereas GPS baseline measurement is carried out independently and hence it is free from accumulation of errors. So it is quite evident that GPS is superior to the conventional survey methods in terms of accuracy. This factor will be much dominant while transforming GPS surveyed WGS-84 coordinate to the local coordinate system. Should the GPS values be distorted by performing an adjustment in which the conventional control is held fixed? Furthermore, currently we are not in a position to determine transformation parameter for whole country rather we are determining "Localized" parameter. Such separate "Localized" parameters for different areas are resulting dual coordinate values in marginal areas. These problems being faced by Survey Department presently must be addressed.

Table - 1 ( Triangular error table)

\begin{tabular}{|c|c|c|c|c|c|}
\hline Series & $\begin{array}{c}\text { Instrument } \\
\text { used }\end{array}$ & $\begin{array}{c}\text { AverageTriangular } \\
\text { error(arc seconds) }\end{array}$ & $\begin{array}{c}\text { MaximumTriangular } \\
\text { error (arcseconds) }\end{array}$ & $\begin{array}{c}\text { Closing error } \\
\text { On Base }\end{array}$ & Remarks \\
\hline A & $\begin{array}{c}\text { 1arc sec } \\
\text { Theodolite }\end{array}$ & 3 & 8 & $1 / 42000$ & Adjusted series \\
\hline B & do & 5 & 17 & $1 / 15000$ & do \\
\hline C & do & 4 & 12 & $1 / 5000$ & do \\
\hline D & do & 4 & 13 & $1 / 12000$ & do \\
\hline F(main) & do & 3 & 10 & $1 / 14000$ & $\begin{array}{c}\text { Unadjusted (closing error not } \\
\text { adjusted) }\end{array}$ \\
\hline E(a) & do & 4 & 10 & - & do \\
\hline E(b) & do & 4 & 10 & $1 / 10000$ & do \\
\hline E(c) & do & 5 & 10 & - & Unadjusted Series \\
\hline f(sub) & do & 3 & 16 & - & do \\
\hline
\end{tabular}


Given the inconsistencies in the local networks a revision by way of a total readjustment is necessary. In order to derive a single set of transformation parameter for the whole country first of all various higher order networks set up at different periods by different parties should be integrated as a single network. The existing triangulation may be referred to as the old network. A new integrated control network comprising all series of existing higher order triangulation stations and different series of GPS stations has been designed and referred to as the Nepal GPS Network. Its coordinates in the WGS-84 reference frame are being determined using GPS survey.

\section{Conclusion}

Moresophisticated techniques and more amount of GPS data are required if one wishes to get sub-meter transformation accuracy. Geodetic Branch of Survey Department has been running the programme of strengthening the Geodetic network since a couple of years and expected to be completed this year. The best co-ordinate value in WGS 84 reference system for Nagarkot station has to be fixed as a reference coordinate for the entire network solution. If we would like to establish the new geodetic network within a global reference frame, i.e., WGS84, it is necessary that the new geodetic network be tied to points within the IGS global network of points with WGS84 coordinates. Two or perhaps three would be sufficient to make the connection to the Global IGS Network. Thus derived parameters will be suited for different applications. The absence of a Geoid computed with respect to the local datum has presented some difficulties in properly analyzing the quality of the height transformations. When these transformations are to be applied in practice, geoid heights will be required. It is therefore necessary that an accurate local geoidal model be developed. Finally, the proposed Nepal GPS Network can be expected to serve as a foundation for any future national control activities, be it a revision of the existing network or a redefinition of the datum.

\section{References:}

1. Acharya P., Adiga G., Geodetic Datum of Nepal, Nepal Surveyors Society Journal, 1992 and Phuyal B.P.

2. Kalyan Gopal Shrestha Unpublished report on $A$ Status Report on GPS Data Processing, 1998

3. Niraj Manandhar and Maheswar P. Bhattarai An overview on Time Series of Geodetic and GPS Network of Nepal, Nepalese Journal on Geoinformatics, 2002
4. S.K.Singh Coordinate transformation between Everest and WGS - 84 datum - A parametric approach, GIS development, 2002

5. Survey Department Topographical Base Map souvenir, Souvenir publication, 2002

6. Survey Department Unpublished report on first order geodetic network of Nepal 1981-1984

7. Survey Department Unpublished report on GPS survey of Eastern and Western Nepal Topographical Mapping Project 1994, 1997.

8. Tirtha B. Pradhananga Surveying and Mapping in Nepalese Context, Nepalese Journal on Geoinformatics, 2007 